\title{
A New Method for Extracting Elevation Information of River System from Contours
}

\author{
He Guifang a , Liu Minshi ${ }^{\mathrm{a}, \mathrm{b}, *}$ \\ a School of Geographic Information and Tourism, Chuzhou University, Chuzhou, China, heguifang1983@126.com \\ b School of Geography Science, Nanjing Normal University, Nanjing, China; liuminshi1983@126.com \\ * Corresponding author: liuminshi1983@126com; Tel.: +86-138-6690-0791
}

\begin{abstract}
Considering the existing problems in methods of extracting elevation in river system, such as low efficiency, low accuracy, elevation logic errors and other issues, this paper proposes a new method of elevation extraction based on contours. Firstly, the spatial relationship between river system and contours is constructed to improve the intersection efficiency. Secondly, the accuracy of river system elevation can be improved and river flow logical error can be avoided by using the method of interpolation of trend surface of the river section. And then, the river tree is established to avoid the logical error of river system. Many comparison experiments about efficiency, elevation accuracy and elevation logical error between this method and other two conventional methods have been carried out, and the results show that both accuracy and efficiency can be improved and there is no logical error of elevation by this method.
\end{abstract}

Keywords: river system, elevation information, contour, spatial relationship

\section{Introduction}

The spatial morphology characteristics of the river system play an important role in watershed geomorphology and terrain analysis as well as natural disaster researches, such as flood, landslide and mud-rock flow (Zhang and Guilbert, 2013). As rivers have three-dimensional spatial features in nature, analyzing the river system on the basis of threedimensional features improves data processing accuracy and produces more objective and scientific conclusions of the spatial morphology of the river system (Liu et al, 2016). However, river system data was preciously presented in Digital Line Graphic (DLG) format after digitalization in the basic geographic spatial database, without elevation information. For this reason, river system elevation extraction is the basis and precondition of river system morphology feature analysis and the study of related fields. Considering the consistency between flow direction and drainage, the river elevation can be extracted based on terrain data corresponding to the river, such as contours and DEM data. The river elevation value can be obtained by interpolation. However, the existing terrain data interpolation methods have disadvantages, such as low efficiency, low accuracy, incorrect river flow direction, inconsistent topological relationship and so on. The piecewise interpolation method requires a great number of intersection operations to calculate river elevation (Huang, 2010). The disadvantages of terrain interpolation and calculating river elevation after DEM interpolation are low accuracy and unwarrantable correct flow direction. Deterministic Eight Neighbors (D8) and its improved method have been proposed to extract drainage network data directly from DEM, using slope runoff to simulate the flow of overland runoff (O'Callaghan and Mark, 1984; Martz and De Jong, 1988; Garbrecht and Martz, 1997). Although the method is relatively fast, the extracted results are obviously different from the real river data.

Therefore, through analyzing the spatial structure relationship between river and contours and establishing correlation, we proposed an improved piece-wise interpolation method that can improve calculation efficiency and accuracy. Meanwhile this method can ensure correct flow directions and topological consistency of rivers in the river system.

\section{Related works}

Currently, the river system elevation extraction methods can be grouped into three categories: piece-wise interpolation based on contours, scattered points interpolation based on the surrounding terrain of river, and interpolation based on DEM.

\subsection{Piece-wise interpolation method based on contours}

The piece-wise interpolation method based on contours is utilized by calculating the intersections of rivers and contours. Then segment the river at the intersection. There are three main steps:(1) Calculate the intersection of rivers and contours, this process can be converted into calculating the intersections between multi-line and multiline. The main drawback of this method is low efficiency. Even if the method of Raster Detection Plus Vector Calculation is used to speed up the calculation, the time 
efficiency can be increased by only about 10-30 times (Fei and $\mathrm{Li}, 1993$ ). Considering the large volume of contours, the efficiency is not enough. (2) Piece-wise river interpolation: piece-wise river interpolation is a linear interpolation in nature. A simpler approach is to assume that the elevation decreasing velocity is always equal on the segmented path of the river and then to linearly interpolate with a specific ratio. This ratio is calculated by $\frac{B}{A}$, where A is the length of the segment, and B is the length from the point being calculated to the point where the elevation is the smaller of the two ends of the segment. (3) Calculate the elevation of the source and the junction of river: as the source and the junction of river are a precondition of a segmented river, the value can be interpolated by the elevation points around.

\subsection{Scatted points interpolation method based on} terrain around the river

Points on the contour lines are regarded as terrain scatters in this method. With terrain scatters being determined by setting the scope of the domain, the points at the river can be interpolated one by one. This method is essentially terrain data interpolation. Commonly used terrain interpolation methods include Inverse Distance Weighted (IDW), Kriging, Local Polynomial, Radial Basis Functions method (RBF), Spline function, ANUDEM and so on (Arun, 2013; Tan, 2014). The disadvantage of this kind of methods is that the accuracy of interpolation is influenced by many factors. For example, different methods are adopted in different terrains and sampling densities; thus, the optimized interpolation to apply to arbitrary conditions is hard to determine. In addition, this method cannot ensure that the flaw direction is absolutely correct in logic.

\subsection{Interpolation method based on DEM}

In this method, the elevation of the river point is obtained by overlapping river data and grid DEM data and then calculating the grid cell which the river point exists (Huang, 2009). As easy as this method is, the accuracy of the extracted drainage is dependent upon the accuracy of DEM, which is influenced by the size of spatial resolution, terrain complexity and many other factors (Li, 1993; Veregin, 1997; Gao, 1997; Aguilar et al, 2005). In addition, the efficiency of obtaining DEM through contours should be taken into account when using this method. Considering that the method cannot guarantee absolutely correct flow direction logic, the elevation value of the river should be readjusted after interpolation to satisfy the flow direction (Grimaldi et al, 2004).

\section{Relationship between river system and contours}

The flow direction tends to coincide with valleys. Starting from the source, flowing along the flow direction until the junction of the river, the elevation of contours that are intersected with the river decreases monotonically with the contour interval as the unit (equal height), as shown in Figure 1. A river system consists of many rivers flowing through many valleys. These valleys form a valley system, which in nature is a valley in a larger spatial scale. Therefore, for the rivers in the same system, the contour line formed by the points at the rivers with the same elevation value should be continuous, and the rivers with the same elevation should intersect the same contour. As shown in Figure 1, all tributary points in the river system with the same elevation value between the mainstream junctions of the river system to 1200 are intersected by the same contour.

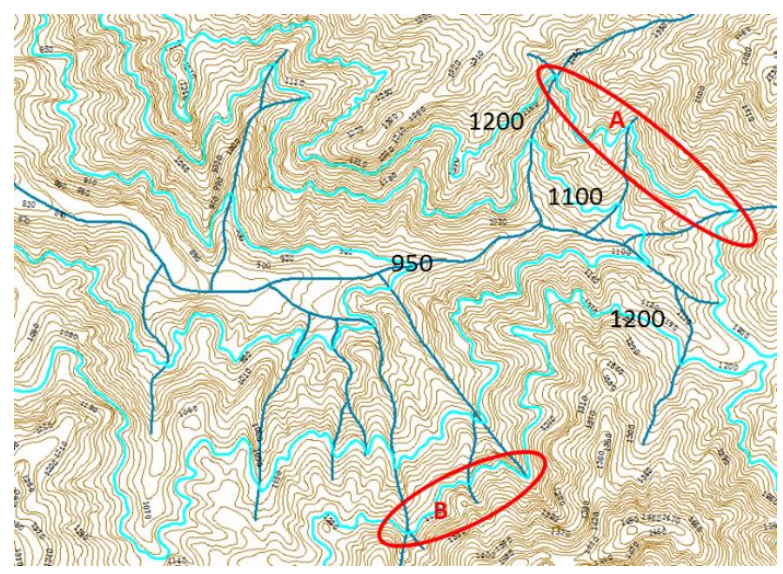

Figure 1. Process diagram of relationship and intersection between river system and contours.

Certainly, the relationship between the river system and the contours is not always established as mentioned above. The relationship cannot be established if (1) the river originated from an independent mountain, and the contour itself is closed and distinguished from others, or (2) map sheet division exists, in which case the contour line is divided, resulting in one completed contour line divided into multiple contours. As shown in Figure 1, the rivers at $\mathrm{A}$ and $\mathrm{B}$ are in the same river network, and their elevation values are the same, but they are not in the same contour line.

Based on this finding, two rules have been proposed as follows.

Rule 1: Flow the river, from a source to a junction, the elevation value of the contours intersected by the river in turn is monotonically decreasing with the contour interval. Setting the contour interval as $d$, the elevation value of the contour intersected by the river as $Z_{i}$, and the elevation value of the next contour intersected by the river as $Z_{j}$, then $Z_{j}=Z_{i}-\mathrm{d}$.

Rule 2: In the same river system, if river $R_{i}$ is intersected with contour $C_{k}$ whose value of elevation is $Z$, and another river $R_{j}$ is intersected with a contour whose value of elevation is $Z$ too. Then $R_{j}$ and $R_{i}$ are both intersected with $C_{k}$.

Description: Abnormal condition exists in Rule 2, and the solution is discussed in 4.3. 


\section{An improved piece-wise interpolation method of extracting the elevation of the river system}

\subsection{Basic idea}

The piece-wise interpolation method based on contours has two inherent disadvantages: (1) low efficiency in obtaining intersections of river and contours, and (2) low accuracy in obtaining elevation of the source and the junction of the river. For this reason, firstly we build a river tree and take the spatial relationship between the river system and contours into account to improve the efficiency in obtaining intersections of rivers and contours. Secondly, we use the established river tree to improve the accuracy of the calculation of the junction elevation and ensure the topological consistency of the elevation of the intersections of rivers. Finally, we use pipeline interpolation to calculate the elevation value of segmented river. At the same time, we use extrapolation and inverse distance weighted interpolation to calculate the junction elevation of the mainstream and the source elevation of river respectively. Accuracy has been improved based on this method.

\subsection{Establish a river tree of the river system}

The key to establishing the river tree is to set up the relationship between the mainstream and the tributary. An easy and effective method that can establish tree structure quickly by searching the tributaries automatically and establishing the buffer of the mainstream has been proposed (Wu, 1995). However, the mainstream cannot be distinguished, which influences the full automaticity of the algorithm. Furthermore, the information of the flow direction and junctions of rivers, which is the basis for subsequent river elevation extraction, cannot be provided. For these reasons, based on the method above, we add three processes: (1) searching of the mainstream, (2) inferring of the flow direction, and (3) searching of the junction of the river. The specific description is as follows.

(1) Searching of the mainstream: The mainstream is a river that does not flow into any other rivers. Therefore, if the two endpoints of a river are not on any other rivers, it is the mainstream (such as river 1 shown in Figure 2). We can determine the mainstream by considering whether the two endpoints are located on any other rivers.

(2) Inferring the flow direction: for each tributary, there must be one endpoint (junction point) located on another mainstream. In this way, the flow direction of tributary can be determined quickly after determining the junction of the river. Up to the mainstream, the elevations of two endpoints must be interpolated by contours, then inferring the flow direction by elevations of two endpoints.

(3) Searching of the junction of the rivers: River junction is the point where the mainstream coincides with the junction of the tributaries. Therefore, from the mainstream, we can search for corresponding junctions of tributaries in turn. If the search is successful then we can record the values; otherwise, we can add the point firstly and then record the values. A schematic diagram of the river tree is shown in Figure 2(a). Numbers 1-5 means river ID, letters $A_{i}, B_{i}$, $C_{i}, \quad \mathrm{D}_{i}, \quad \mathrm{E}_{i}(i=0,1,2, \ldots)$ represent a series of points on the rivers, and letter subscripts represent the order of river data storage. Figure 2(b) is the schematic diagram of the river tree based on Figure 2(a). In Figure 2(b), each node includes 4-tuples :( 1) River ID; (2) river junction; (3) river direction (if the direction is consistent with data storage direction, note T; otherwise, note F); and (4) the index of the junction recorded in the mainstream, and -1 means that the mainstream is not exist

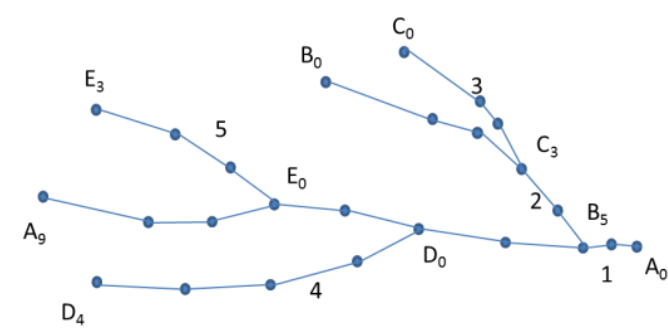

(a)

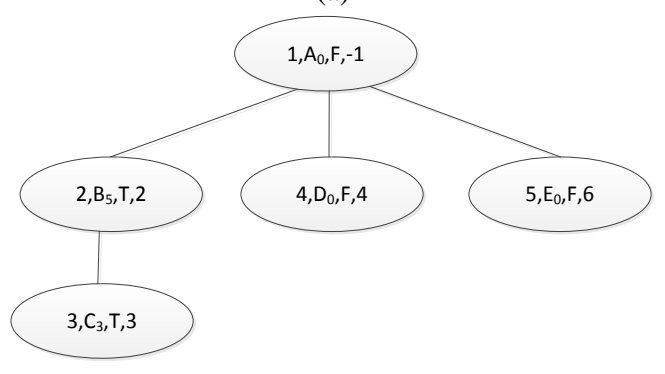

(b)

Figure 2. Schematic diagram of river tree. They should be listed as: (a) Schematic diagram of river system; (b) Schematic diagram of tree structure.

\subsection{Obtaining of intersections quickly based on the} relationship between river system and contours

Definition1: Active contour collection (ACS): the collection stores elevation-ordered (increased by contour interval) contours, which are intersected with the rivers. At the same time, only one contour value can be stored when multiple contours have the same elevation. The collection simplifies the contour searching process when calculating the intersections of the contours and the rivers.

Rules 1 and 2 are used to speed up the process of obtaining intersections based on the relationship between river system and contours. According to Rule 1, the elevation of the contours that intersect with the river can be determined in sequence only if the junction elevation of the river is known. In this way, the efficiency of the contour searching is improved. According to Rule 2, the search scope of contours for the same river system can be determined quickly by using collection ACS. Considering that the junction elevation of the tributary is obtained from the mainstream, the established ricer tree is depth-first traversed to prevent disposing the tributaries before the mainstream. The method of dealing with the mainstream is different from the others, as it is the first one to dispose. 
The mainstream is treated as follows:

Step1. Initialize the elevation of the source $\left(Z_{o p}\right)$ and the junction $\left(\mathrm{Z}_{s p}\right)$ of the river. $\mathrm{Z}_{o p}$ and $\mathrm{Z}_{s p}$ are obtained by nearest-neighbor interpolation. The values of $\mathrm{Z}_{o p}$ and $\mathrm{Z}_{s p}$ are equal to the values of the nearest contours.

Step2. Set the junction of the river as the initial position and calculate the value of contours $\left(Z_{i}\right)$ that intersect with the mainstream. If it is the first one, then $Z_{i}=\mathrm{Z}_{s p}$ or $Z_{i}=\mathrm{Z}_{s p}+d$; otherwise, $Z_{i}=$ $Z_{i}+d . d$ is the contour interval.

Step3. Search contours. Find contours, intersecting with rivers, whose value is $Z_{i}$ by traversing the contour collection. If the search is successful and $Z_{i}$ is more than $Z_{\text {max }}$ in ACS, then add it into ACS; otherwise, end the program.

Step4. Obtain the intersections of the river and the contours, and then intersect them in the corresponding position of the river and turn to step 2 .

The other rivers are treated as follows:

Step1. Initialize the elevation of the source $\left(Z_{o p}\right)$ and the junction $\left(\mathrm{Z}_{s p}\right)$ of the river. $\mathrm{Z}_{s p}$ can be obtained by the intersection of the river and its "parent river", according to the river tree. The approach to obtain $\mathrm{Z}_{o p}$ is consistent with the approach to obtain $\mathrm{Z}_{o p}$ of the mainstream.

Step2. Set the junction of the river as the initial position and calculate the value of contours $\left(Z_{i}\right)$ that intersect with the mainstream. If it is the first one, then $Z_{i}=\operatorname{Ceil}\left(Z_{s p} / d\right) \times d$; otherwise, $Z_{i}=$ $Z_{i}+d$. Ceil is a roundup function, and $\mathrm{d}$ is the contour interval.

Step3. Search contours. Find contours, intersecting with rivers, whose value is $Z_{i}$ by traversing ACS. If the search is successful, turn to step 5; otherwise, turn to step 4.

Step4. Exception handling. Calculate the difference between the elevation value of $\mathrm{Z}_{o p}$ and $Z_{i}$, and if $\mathrm{Z}_{\text {op }}-Z_{i}<d$, then exit; otherwise, set $Z_{i}$ as the initial contour elevation and continue to dispose it with the current location as the mainstream. As shown in Figure 3, intersections of river $R_{2}$ and contours are obtained. The ACS of the mainstream $R_{1}$ is $\{1,2,3,4,5,6\}$. When searching contours intersecting with $\mathrm{R} 2$, there will be an exception on point $\mathrm{O}_{1}$, becourse contour 7 is not in the ACS of $\mathrm{R}_{1}$. In this case, handle from point $O_{1}$ toward the upstream direction as the mainstream. Similarly, intersections of river $R_{3}$ and the contours are obtained, and an exception occurs from contour 9 . In this case, handle from point $\mathrm{O}_{2}$ toward the upstream direction as the mainstream.

Step5. Obtain the intersections of the river and the contours, and then intersect them in the corresponding positions of the river, then turn to step 2.

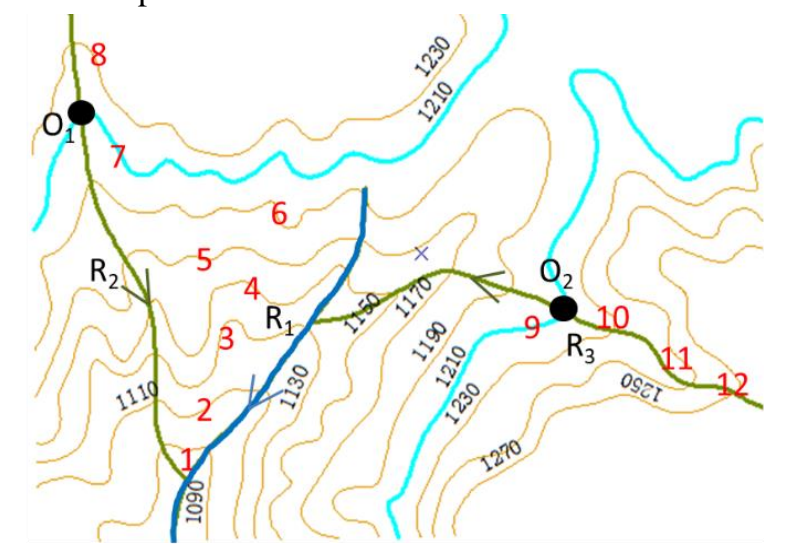

Figure 3 Process diagram of relationship and intersection between river system and contours.

4.4 Piece-wise interpolation of river system and calculation of the source and the junction of the river

The Piece-wise interpolation method, which assumes that the decreasing velocity of the river elevation is the same in the piece-wise path. It is adopted to interpolate linearly with river segment length as the scale. The formula is shown in Formula (1).

$$
z_{i}=\frac{\text { length }\left(p_{i-1}, p_{i}\right)}{\sum_{i=1}^{n} \operatorname{length}\left(p_{i-1}, p_{i}\right)} \times d+z_{0}
$$

Here, length $\left(p_{i-1}, p_{i}\right)$ is the length of adjacent river point (e.g., straight line segment $p_{i-1} p_{i}$ in Figure 4), $d$ is the contour interval, and $z_{0}$ is the elevation value of $p_{0}$.

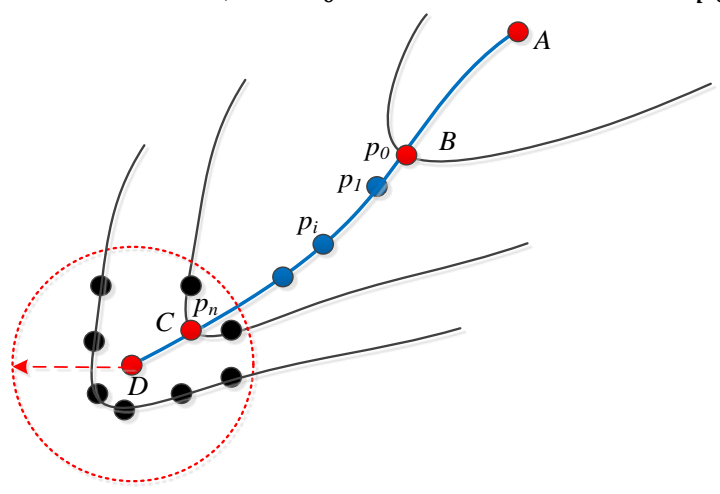

Figure 4. Elevation calculation method of river point. In the figure, black lines are contours, the blue line is river, $\mathrm{A}$ is the junction of the river, $\mathrm{D}$ is the source of the river, $\mathrm{B}$ and $\mathrm{C}$ are intersections of the river and contours, and $p_{i}$ is the point string of the river.

With regard to the source of the river (point D in Figure 4), a relatively precise value can be obtained through spatial intersection of the contours, which are relatively dense around this point. Therefore, inverse distance weighted interpolation, which is easy and in common use, is adopted. With regard to the junction of the tributary, the elevation can be projected through line computation of the river point elevation of the mainstream. The junction of the tributary must be intersected with the corresponding intersection of the mainstream. In addition, the depth-first traverse method is adopted to traverse the established tree 
structure of the river system. Therefore, the process of the tributary follows that of the mainstream.

With regard to the junction of the mainstream (as point $\mathrm{A}$ shown in Figure 4), the value can be obtained by extrapolating the adjacent river contours. The sparse contours lead to inadequate and unevenly distributed contour points, and most junction positions are located near the map border. Thus, the method is shown in Formula (2).

$$
Z_{A}=z_{B}-\frac{\text { length }(A, B)}{\text { length }(B, C)} \times d
$$

Whenlength $(A, B) \geq$ length $(B, C)$,

$$
z_{A}=z_{B}-d+\varepsilon
$$

Here, $\varepsilon$ is an infinitesimal positive number.

\section{Experiment Results}

\subsection{Experimental scheme}

Mountain river system data and corresponding contour data of a 1:50000 standard map of Anhui Province (Figure $5)$ are adopted to conduct the experiment. The elevation information of the river system is obtained through normal piece-wise interpolation (NPWI), inverse-distance weighted interpolation (IDWI) and the method proposed in this paper (IPWI). Then, the outcomes were compared from efficiency, accuracy and elevation logic. The inversedistance weight index is 2 , adaptive searching strategy is adopted, and the number of neighborhood points is more than 4 (Lloyd, 2005). The algorithm is implemented through the .NET platform and C\#, and ArcGIS was used for data visual and statistical analysis.

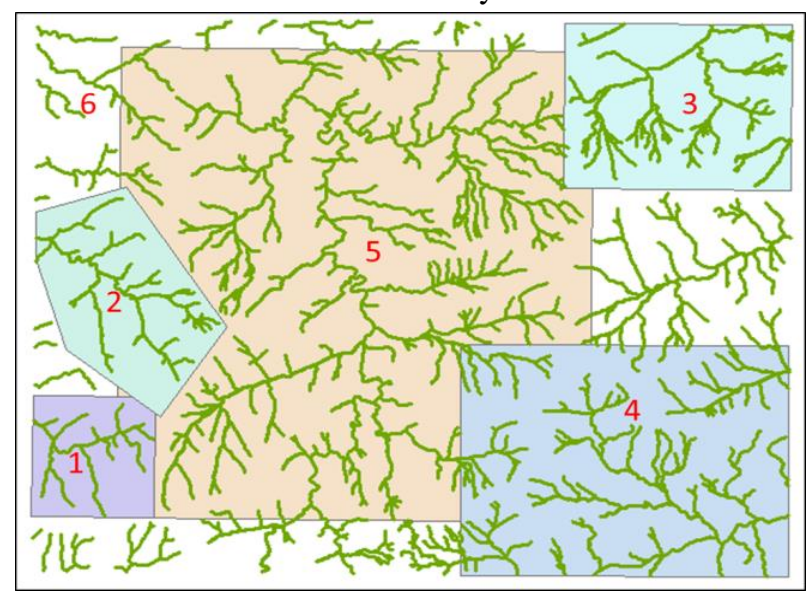

Figure 5. Experimental data of river system.

\subsection{Efficiency analysis}

As shown in Figure 5, data from six different scopes are adopted to increase the reliability of the algorithm efficiency analysis. The configuration of the computer used is a Quad core CPU with $3.4 \mathrm{GHz}$ of dominant frequency and $8 \mathrm{~GB}$ of memory. The run time is shown in table.1. The efficiency of the method proposed in this paper is better than the others. The advantage of IPWI is more obvious with the increase in data volume. This is especially true compared with NPWI, as the efficiency gradually increases from 55 times in data 1 to more than 100 times in data 6 . In addition, the run time of this method for the whole map (data 6) is $10 \mathrm{~min} 35 \mathrm{~s}$, while the others

\begin{tabular}{|c|c|c|c|c|c|}
\hline \multirow[t]{2}{*}{ ID } & \multicolumn{2}{|c|}{ Data Volume } & \multirow[t]{2}{*}{ NPWI } & \multirow[t]{2}{*}{ IDWI } & \multirow[t]{2}{*}{ IPWI } \\
\hline & $\begin{array}{l}\text { Riv- } \\
\text { ers }\end{array}$ & $\begin{array}{l}\text { Con- } \\
\text { tours }\end{array}$ & & & \\
\hline 1 & 12 & 273 & $55 \mathrm{~s}$ & $20 \mathrm{~s}$ & $1 \mathrm{~s}$ \\
\hline 2 & 28 & 463 & $8 \min 41 s$ & $3 \min 1 s$ & $6 s$ \\
\hline 3 & 62 & 699 & $11 \min 1 \mathrm{~s}$ & $\begin{array}{l}3 \mathrm{~min} \\
49 \mathrm{~s}\end{array}$ & $9 \mathrm{~s}$ \\
\hline 4 & 104 & 1051 & $\begin{array}{l}1 \mathrm{~h} 3 \mathrm{~min} \\
12 \mathrm{~s}\end{array}$ & $\begin{array}{l}16 \mathrm{~min} \\
34 \mathrm{~s}\end{array}$ & $25 \mathrm{~s}$ \\
\hline 5 & 301 & 2471 & $\begin{array}{l}7 \mathrm{~h} 24 \mathrm{~min} \\
13 \mathrm{~s}\end{array}$ & $\begin{array}{l}2 \mathrm{~h} \quad 19 \\
\min 41 \mathrm{~s}\end{array}$ & $\begin{array}{l}3 \mathrm{~min} \\
51 \mathrm{~s}\end{array}$ \\
\hline 6 & 535 & 3923 & $>24 \mathrm{~h}$ & $>24 \mathrm{~h}$ & $\begin{array}{l}10 \mathrm{~min} \\
35 \mathrm{~s}\end{array}$ \\
\hline
\end{tabular}
cannot be completed in 24 hours.

Table 1. Contrast of efficiencies among three methods.

\subsection{Accuracy comparison}

The accuracy of extracting river elevation can be obtained by analyzing the elevation error of each river point. The river elevation error formula is as follows:

$$
\mathrm{E}=\frac{1}{n} \sum_{i=1}^{n}\left|Z_{i}-\bar{Z}_{i}\right|
$$

Here, $E$ is the average error, $Z_{i}$ is the river point elevation extracted by the algorithm, $\bar{Z}_{i}$ is the real data, and $n$ is the number of river points. $\bar{Z}_{i}$ can be obtained by artificial extraction.

We extracted the elevation values of rivers in data 1 using three algorithms. Then calculated $\mathrm{E}$ of each river. The outcomes are shown in table. 2. As shown in the table, the error of IDWI is the largest, the error value is between 2.89 6.23m; the errors of NPWI and IPWI are much smaller, most of which are smaller than $1 \mathrm{~m}$. Furthermore, IPWI is better than NPWI because the error is smaller, with the exception of river 1 and river 10 .

\begin{tabular}{|l|l|l|l|l|l|l|l|}
\hline \multirow{2}{*}{ ID } & \multicolumn{3}{|l|}{ Average Error $(\mathrm{m})$} & \multirow{2}{*}{ ID } & \multicolumn{3}{|c|}{ Average Error $(\mathrm{m})$} \\
\cline { 2 - 5 } \cline { 6 - 8 } & $\begin{array}{l}\text { ID- } \\
\text { WI }\end{array}$ & $\begin{array}{l}\text { NP- } \\
\text { WI }\end{array}$ & $\begin{array}{l}\text { IP- } \\
\text { WI }\end{array}$ & & $\begin{array}{l}\text { ID- } \\
\text { WI }\end{array}$ & $\begin{array}{l}\text { NP- } \\
\text { WI }\end{array}$ & $\begin{array}{l}\text { IP- } \\
\text { WI }\end{array}$ \\
\hline 1 & 5.48 & 0.25 & 0.25 & 7 & 6.23 & 0.64 & 0.58 \\
2 & 3.59 & 0.74 & 0.41 & 8 & 2.89 & 0.56 & 0.38 \\
3 & 4.27 & 0.46 & 0.19 & 9 & 2.89 & 1.40 & 0.22 \\
4 & 4.88 & 0.51 & 0.23 & 10 & 3.67 & 0.38 & 0.38 \\
5 & 4.56 & 0.99 & 0.37 & 11 & 2.90 & 0.64 & 0.44 \\
6 & 3.77 & 0.33 & 0.25 & 12 & 5.15 & 0.56 & 0.54 \\
\hline
\end{tabular}

Table 2. Contrast of mean river elevation errors among results using three algorithms with data 1.

\subsection{Elevation logic analysis}

River elevation logic is divided into the logic of flow direction and topological logic of river junction. The former refers to whether the single river elevation value is consistent with the flow direction, or whether "countercurrent" exists. The latter refers to whether the elevation of tributary junction is consistent with the elevation of the mainstream converging point. 
The result of the contrast among river system elevation logic using three methods with data 1 is shown in table. 3 . For the logic of flow directions, IDWI has many errors in each river for a total of 131; NPWI has one mistakes in each of the five rivers; and IPWI is completely correct. The result is confirmed in Figure 6, which describes the contrast among river flow logical consistency using three methods with river 1 . The figure shows that there are multiple "counter-current" errors in IDWI, NPWI has one "counter-current" error at each junction and IPWI has no errors. For the logic of junctions (table. 3), only NPWI has errors. It produces 11 errors in adopting two different interpolations to calculate the elevation for the coincidence of the two points.

\begin{tabular}{|l|l|l|l|}
\hline \multirow{2}{*}{ Elevation Logic Error } & \multicolumn{3}{|c|}{ Number of Errors } \\
\cline { 2 - 4 } & ID- & NP- & IP- \\
& WI & WI & WI \\
\hline Flow Direction Error & 131 & 5 & 0 \\
Intersection Topological Error & 0 & 11 & 0 \\
\hline
\end{tabular}

Table 3. Contrast among river system elevation logic using three methods with dataset 1 .

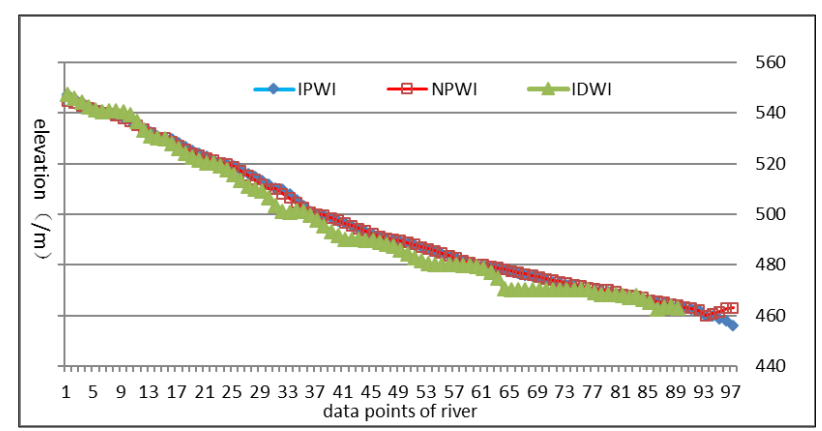

Figure 6. Contrast among river flow logical consistency using three methods with river 1 .

\section{Conclusions}

Elevation information has been extracted through the improved piece-wise interpolation method based on contours. This method builds a river tree structure by analyzing the spatial relationship of the river and contours to establish a method of fast intersection of the river system and contours and a method of river-segmented trend surface interpolation. Compared with the existing water system elevation extraction algorithm, which cannot balance the elevation accuracy and the efficiency of the algorithm, this method not only achieves higher-accuracy water level elevation extraction results but also greatly improves the efficiency of the algorithm. At the same time, it avoids the logic errors of flow direction and the elevation topological errors in the river elevation extraction. Therefore, the accuracy and efficiency of the water system elevation can achieve practical results. The disadvantage of this method is that it can only apply to tree-like drainage. For this reason, it is not applicable to surface water and mesh water systems.

\section{Acknowledgements}

The research introduced in this paper is supported by the National Natural Science Foundation of China (grant No.:
41601499,4150496), and the Key University Natural Science Research Project of Anhui Province, China (Project No.: KJ2017A415). Prof. FEI Lifan is especially appreciated for his generous support and guidance to the research of this topic.

\section{References}

Zhang, L. and Guilbert, E. (2013). Automatic drainage pattern recognition in river system. International Journal of Geographical Information Science, 12, 2319-2342.

Liu, M., Long, Y. and Fei, L. (2016). Line Simplification of Three-dimensional Drainage Considering Topological Consistency. Acta Geodaetica et Cartographica Sinica, 45(4), 494-501.

Huang, L. (2010). Study on the Integrated Generalization of Relief and Water System Based on General DEM. Doctor, Wuhan University, Wuhan, China.

O'Callaghan, J. F. and Mark, D. M. (1984). The Extraction of Drainage Networks from Digital Elevation Data. Computer Vision Graphics \& Image processing, 28(3), 323-344.

Martz, L. W. and De Jong, E. (1988). CATCH: a FORTRAN Program for Measuring Catchment Area from Digital Elevation Models. Computers \& Geoscience, 14(5), 627-640.

Garbrecht, J. and Martz, L. W. (1997). The Assignment of Drainage Direction over Flat Surfaces in Raster Digital Elevation Models. Journal of Hydrology, 193(1), 204213.

Fei, L. and Li, P. (1993). Accelerating the Process of Intersection of Vector Data by Raster Detection Plus Vector Calculation. Acta Geodaetica et Cartographica Sinica, 22(3), 195-204.

Arun, P. V. (2013). A Comparative Analysis of Different DEM Interpolation Methods. Geodezijos Darbai, 39(4):7-13.

Tan Q, Xu X.(2014) Comparative analysis of spatial interpolation methods: an experimental study[J]. Sensors \& Transducers, 165(2): 155.

HUANG Lina, FEI Lifan, HE Jin(2009). Integrated Generalization of Cartographic Symbols for Water System and Geomorphology[C]. Proceeding of the 2nd International Conference on Earth Observation for Global Changes, Vol.1, 2062-2079.

Li, Z. (1993). Mathematical models of the accuracy of digital terrain model surfaces linearly constructed from square gridded data. The Photogrammetric Record, 14(82), 661-674.

Veregin, H. (1997). The effects of vertical error in digital elevation models on the determination of flow-path direction. Cartography and Geographic Information Systems, 24(2), 67-79.

Gao, J. (1997). Resolution and Accuracy of terrain representation by grid DEMs at a micro-scale. International Journal of Geographical Information Science, 11(2), 199-212. 
Aguilar, F. J., Agüera. F., Aguilar, M. A. and Carvajal F. (2005). Effects of terrain morphology, sampling density, and interpolation methods on grid DEM accuracy. Photogrammetric Engineering \& Remote Sensing, 71(7), 805-816.

Grimaldi, S., Teles, V. and Bras, R. L. (2004). Sensitivity of a Physically Based Method for Terrain Interpolation to Initial Conditions and its Conditioning on Stream Location. Earth Surface Processes and Landforms, 29(5), 587-597.
Wu, H. (1995). Automatic Establishment of River Tree Structure. Journal of the Wuhan Technical University of Surveying and Mapping, s, 7-14.

Lloyd, C, D. (2005). Assessing the effect of integrating elevation data into the estimation of monthly precipitation in Great Britain. Journal of Hydrology, 308(1), 128-150. 